\title{
A phase II clinical trial of polyethylene glycol-conjugated L-asparaginase in patients with advanced ovarian cancer: Early closure for safety
}

\author{
JOHN L. HAYS ${ }^{1}$, GEOFF KIM ${ }^{1}$, AMANDA WALKER ${ }^{1}$, CHRISTINA M. ANNUNZIATA ${ }^{1}$, JUNG-MIN LEE ${ }^{1}$, \\ JENNIFER SQUIRES ${ }^{1}$, NICOLE HOUSTON ${ }^{1}$, SETH M. STEINBERG ${ }^{2}$ and ELISE C. KOHN ${ }^{1}$ \\ ${ }^{1}$ Women's Cancers Clinic, Medical Oncology Branch, Center for Cancer Research, National Cancer Institute, \\ Bethesda, MD 20892; ${ }^{2}$ Biostatistics and Data Management Section, Center for Cancer Research, \\ National Cancer Institute, Rockville, MD 20852, USA
}

Received February 5, 2013; Accepted March 1, 2013

DOI: $10.3892 / \mathrm{mco} .2013 .99$

\begin{abstract}
The anti-angiogenic activity of L-asparaginase (L-ASP) and the sensitivity of ovarian cancer cell lines to L-ASP has been previously demonstrated by preclinical findings. The aim of this clinical trial was to translate those findings and evaluate the activity of polyethylene glycol-conjugated L-asparaginase (PEG-ASP or pegaspargase) in advanced ovarian cancer. Women with recurrent ovarian cancer and good end-organ function were enrolled in an open-label phase II trial of PEG-ASP at a dose of $2,000 \mathrm{IU} / \mathrm{m}^{2}$ by intravenous infusion every 2 weeks. Patients were evaluated for response every 8 weeks and for toxicity on an ongoing basis. Early stopping rules for toxicity and activity were included. Four patients were enrolled and received a total of 7 treatment cycles. The study ended accrual by invoking an early stopping rule, after excessive toxicity was identified in patients. Drug-related toxicities included grade 2 pancreatitis, fatigue, neutropenia, hypoalbuminemia, weight loss, dehydration, decreased fibrinogen and 1 case of grade 3 hypersensitivity reaction during cycle 2 . One patient died during the study. No patients were evaluable for response. PEG-ASP was poorly tolerated in this group of advanced-stage ovarian cancer patients and no conclusions regarding activity may be drawn. Further studies of PEG-ASP in ovarian cancer patients are not recommended.
\end{abstract}

\section{Introduction}

There is an unmet need to develop effective and novel treatment strategies for epithelial ovarian cancer (EOC), which is

Correspondence to: Dr Elise C. Kohn, Women's Cancers Clinic, Medical Oncology Branch, Center for Cancer Research, National Cancer Institute, 9000 Rockville Pike, Building 10, Room 12N226, Bethesda, MD 20892, USA

E-mail: kohne@mail.nih.gov

Key words: ovarian cancer, pegaspargase, L-asparaginase, angiogenesis the leading cause of mortality due to gynecological malignancies in the United States of America (1). The majority of patients present with advanced-stage disease (stage III/IV), for which cure is rare, with a 5-year median survival rate of $<20 \%$ for stage IV patients (1). A possible method of augmenting the therapeutic armamentarium is reassessing and repurposing previously examined agents.

Targeted molecular therapies against cancer cells and surrounding stromal cells (fibroblasts, endothelial and inflammatory cells) are being developed as the signaling pathways that drive tumor development and progression are elucidated. Stromal cells permeate the tumor microenvironment and play a critical role in cancer development, growth, invasion and metastasis (2). EOCs induce extensive neovascularization which facilitates tumor growth and metastasis. We previously demonstrated that increased vasculature correlates with higher-grade EOC tumors and poor survival (3). Numerous therapies have been developed to target the interactions between tumor cells and the stroma, including anti-angiogenic agents, exemplified by bevacizumab, a monoclonal antibody against vascular endothelial growth factor (VEGF) that has some single-agent phase II clinical activity in platinum-resistant EOCs (4). However, this and other biological agents have not significantly extended the progression-free or overall survival in EOC. Therefore, there is increasing interest in therapies that exhibit antistromal as well as antitumor activity.

We hypothesized that L-asparaginase (L-ASP) may be categorized as an agent capable of targeting the tumor as well as the tumor microenvironment through its antimetabolic activity. L-ASP has been the mainstay of treatment of patients with acute lymphoblastic leukemia for $>40$ years $(5,6)$. Treatment with L-ASP leads to depletion of circulating L-asparagine in the serum of patients. It has been argued that this depletion may cause injury to tumor cells that are not capable of synthesizing adequate amounts of this amino acid to meet the needs of rapid cell division $(7,8)$. Lorenzi et al demonstrated a negative association between the frequently low asparagine synthase (ASNS) transcript and protein levels in ovarian cancer cell lines with sensitivity to L-ASP. This led 
us to consider L-ASP as a potential therapeutic agent to repurpose for EOC $(9,10)$. Taylor et al demonstrated the efficacy of polyethylene glycol-conjugated L-asparaginase (PEG-ASP or pegaspargase) on fresh ovarian cancer tumor specimens using a human tissue clonogenic assay (11). We recently demonstrated that L-ASP inhibits capillary-like tube formation of human microvascular endothelial cells (HMVECs) and decreases heterotypic adhesion between ovarian cancer and endothelial cells. This occurs at concentrations that exert no direct antiproliferative effect on HMVECs and limited antiproliferative effects on ovarian cancer cells (12). These data suggest that L-ASP exhibits activity against EOC, which is independent of proliferation pathways.

PEG-ASP, which is administered intravenously (i.v.) or intramuscularly (i.m.), was developed due to the high rates of allergic reactions and the need for frequent i.m. injections with its non-conjugated form, L-ASP (13). Ho et al investigated the clinical pharmacology of PEG-ASP in 27 adults, at doses of $500-8,000 \mathrm{U} / \mathrm{m}^{2}$ administered i.v. over $60 \mathrm{~min}$ (14). In this early study, i.v. PEG-ASP was determined to have a half-life of $357 \mathrm{~h}$ (14.8 days), thus supporting the use of every-2-week treatment. A previous phase I dose-escalation study using PEG-ASP in 28 adult patients with advanced solid tumors reported no treatment-related patient mortality and only 1 case of grade 4 toxicity (allergic reaction) (11). Our clinical trial was designed with the aim to translate preclinical findings into clinical practice and evaluate the efficacy of PEG-ASP in patients with advanced ovarian cancer.

\section{Materials and methods}

Patient selection. Patient eligibility criteria included persistent, relapsed or refractory epithelial ovarian, fallopian tube, or primary peritoneal cancer, measurable disease according to the Response Evaluation Criteria in Solid Tumors (RECIST v1.0), a minimum 4-week washout period and no limit regarding the number of previous regimens. All the patients had Eastern Cooperative Oncology Group (ECOG) performance status $\leq 2$. Adequate end-organ function was defined as follows: absolute neutrophil count (ANC) $>1500 / \mathrm{mm}^{3}$; platelet count $>100,000 / \mathrm{mm}^{3}$; serum creatinine $\leq 1.5 \mathrm{mg} / \mathrm{dl}$, or if low, creatinine clearance $\geq 60 \mathrm{ml} / \mathrm{min}$; total bilirubin $\leq 1.5 \mathrm{x}$ upper limit of normal (ULN), unless in the case of Gilbert's syndrome; lipase and amylase $\leq 1.5 \times \mathrm{ULN}$; aspartate aminotransferase (AST) and alanine aminotranferase (ALT) $\leq 2.5 \times$ ULN; fibrinogen $\geq 0.75 \times$ lower limit of normal; and prothrombin time (PT), partial thromboplastin time (PTT) and international normalized ratio (INR) $\leq 1.5 \times$ ULN.

Patients were required to have recovered from any prior treatment-related toxicity to CTCAE v3.0 grade 1, except for stable peripheral neuropathy, from which they were required to have recovered to grade 2 or lower (alopecia and hypertension were exempted). Patients were excluded if there was evidence of a severe or uncontrolled concurrent condition, brain metastases, history of clinically symptomatic pancreatitis within the past 6 months, deep venous thrombosis (DVT) within the past 3 months or pulmonary embolism within the past 6 months, history of recurrent thrombosis or pulmonary embolism, requirement for ongoing full-dose anticoagulation, or prior exposure to any formulation of L-ASP. Another prerequisite was the presence of biopsiable disease or fluid collections that could be sampled for research purposes. This study was approved by the Institutional Review Board of the National Cancer Institute and all patients provided written informed consent prior to study enrollment, in compliance with institutional, state and federal regulations.

Study design and drug administration. This was a non-randomized, open-label, investigator-initiated study, aiming to assess the activity and toxicity of i.v. pegaspargase. Pegaspargase was provided by Sigma-Tau Pharmaceuticals, Inc. (Gaithersburg, MD, USA), under a Clinical Trials Agreement with the National Cancer Institute. This study was designed as a two-stage optimal design (15) in order to evaluate whether a sufficiently high proportion of patients were able to attain a 6-month progression-free interval (PFI) using pegaspargase. Patients were evaluated by history and physical examination every 2 weeks during the first cycle and at least every 4 weeks thereafter. Complete blood count (CBC) with differential and platelet count, peripheral blood PT/activated partial thromboplastin time (aPTT) and fibrinogen, amylase and lipase, metabolic panel including liver enzymes and cancer antigen 125 (CA-125) were determined with each cycle. Patients received i.v. pegaspargase of 2,000 IU/m $\mathrm{m}^{2}$ every 2 weeks, with each cycle consisting of 2 doses. Reassessment computed tomography (CT) imaging to document response was performed every 2 cycles. The drug was discontinued in the case of grade 3 allergic reaction or other drug-limiting toxicity, cancer progression, other intercurrent medical issues, or at the request of the patient. Patients who achieved a complete response were scheduled to receive a further 2 cycles.

Dose modifications. Dose adjustments were made as follows: Treatment was interrupted in the case of CTCAE grade 2 or higher adverse events (AE) deemed attributable to pegaspargase. Dose was reduced to $1,500 \mathrm{IU} / \mathrm{m}^{2}$ in the case of a repeat grade 2 event recovering within 3 weeks. Delay beyond 3 weeks resulted in discontinuation of the treatment. A first grade 3 or hematological grade 4 event, recovering to grade 1 within 3 weeks resulted in dose reduction to $1,500 \mathrm{IU} / \mathrm{m}^{2}$. Any non-hematological grade 4 event, clotting event (thrombosis or embolism), grade 2 or higher coagulopathy, grade 2 or higher allergic reaction and grade 3 or higher pancreatitis necessitated discontinuation of the treatment. No dose adjustments were made for grade 2 hypertension, alopecia or neuropathy or for any suboptimally managed grade 2 or 3 nausea.

Response criteria. Response was evaluated according to RECIST v1.0 (16). The effect of pegaspargase on CA-125 production and secretion is unknown; therefore, CA-125 was not used as a measure of response.

Statistical analysis. This study was designed with a two-stage optimal design (15) to evaluate the proportion of patients attaining a 6-month PFI. A PFI that potentially improves upon the findings of the GOG 0126 series was defined as the target objective (17). The GOG 0126 series included similar patients with platinum-resistant disease and reported an overall PFI of $16 \%$, with a $95 \%$ confidence interval (CI) width of $\pm 5 \%$. 
Using $\alpha=0.10$ and $\beta=0.10$, this trial targeted $31 \%$ of patients to be progression-free at or beyond 6 months $(\mathrm{p} 1=0.31)$ and was designed to rule out an $11 \%$ probability of attaining a 6-month PFI ( $\mathrm{p} 0=0.11$ ), which corresponded to the lower $95 \%$ $\mathrm{CI}$ on the $16 \%$ rate obtained in the GOG 0126 series. Thirteen evaluable patients were to be enrolled initially, with 2 out of the 13 passing the 6-month PFI required to continue the full accrual of 30 patients.

An early stopping rule for excessive toxicity was incorporated in the study: if 2 out of the first 6 patients developed any severe AE or a clotting event attributable to the agent, no further patients would be enrolled, since the upper $90 \%$ one-sided CI bound for 2 patients with severe AEs within 6 patients is $66.7 \%$, consistent with an excessive rate of toxicity. If at any time beyond the first 6 patients, the fraction of patients experiencing a severe AE exceeding 1/3, accrual to the trial would be halted and trial continuation reassessed.

\section{Results}

Patient characteristics and tumor response. Four patients with high-grade serous ovarian cancer were treated (Table I). All the patients had received multiple prior regimens of cytotoxic chemotherapy (median, 8) and had pelvic disease. Three patients had metastatic liver disease and 1 had metastasis in the pancreas. A total of 8 doses of medication were administered to the 4 enrolled patients. One patient received 3 doses, 2 patients received 2 doses and 1 patient received only 1 scheduled dose of PEG-ASP. No patient was able to receive the full 2-cycle treatment and on-time response reassessment. No patients exhibited clinical or radiographic evidence of disease progression during the study.

Toxicities. Trial enrollment was discontinued after 4 patients, following the early stopping rule for excessive toxicity. All toxicities grade $\geq 2$ are listed in Table II. One patient exhibited a grade 3 allergic reaction during administration of her third dose of pegaspargase. The patient had received premedication with $10 \mathrm{mg}$ dexamethasone, $16 \mathrm{mg}$ ondansetron, $25 \mathrm{mg}$ diphenhydramine and $50 \mathrm{mg}$ ranitidine prior to initiation of the infusion with pegaspargase. The patient reported retro-orbital pressure, pruritis and vomiting within $30 \mathrm{~min}$ of starting the infusion. The infusion was interrupted and she was administered $50 \mathrm{mg}$ diphenhydramine, $2 \mathrm{mg}$ lorazepam and $100 \mathrm{mg}$ hydrocortisone. Symptoms resolved within $20 \mathrm{~min}$ and the infusion was restarted. Within $15 \mathrm{~min}$, she reported disseminated pruritis, burning sensation in her extremities and retro-orbital pressure and was found to have new-onset tachycardia ( $>110 \mathrm{bpm})$. The infusion was again discontinued with prompt resolution of symptoms and the patient was not administered any more pegaspargase.

One patient exhibited persistently decreased plasma fibrinogen concentration (grade 2), necessitating study drug discontinuation. A third patient withdrew informed consent citing poor quality of life following completion of the first cycle; she experienced persistent nausea, early satiety and abdominal pain, attributed to pancreatitis (with persistent grade 2 elevated lipase and normal amylase levels). This patient had a 1.2-cm metastasis in her pancreas with no labora-
Table I. Patient characteristics and tumor response.

\begin{tabular}{lc}
\hline Patient characteristics & Patient no. \\
\hline Median age (range), in years & $54(35-65)$ \\
ECOG performance status & \\
0 & 0 \\
1 & 3 \\
2 & 1 \\
Stage & 3 \\
IIIC & 1 \\
IV & \\
Histological subtype & 4 \\
Serous & \\
No. of previous therapies & 8 \\
Median & $6-10$ \\
Range & \\
\hline
\end{tabular}

ECOG, Eastern Cooperative Oncology Group.

Table II. Adverse events.

\begin{tabular}{|c|c|c|c|c|}
\hline \multirow[b]{2}{*}{ Toxicity } & \multicolumn{4}{|c|}{ Grade (no. of patients) } \\
\hline & 2 & 3 & 4 & 5 \\
\hline Increased alkaline phosphatase & 1 & & & \\
\hline Hypoalbuminemia & 2 & & & \\
\hline Weight loss & 1 & & & \\
\hline Decreased fibrinogen & 1 & & & \\
\hline Anorexia & 1 & & & \\
\hline Dehydration & 1 & & & \\
\hline Decreased ANC & 1 & & & \\
\hline Thromboembolic event & & & & \\
\hline Allergic reaction & & 1 & & \\
\hline Pancreatitis & 1 & & & \\
\hline Elevated lipase & 1 & & & \\
\hline Elevated amylase & 1 & & & \\
\hline
\end{tabular}

ANC, absolute neutrophil count.

tory findings or clinical symptoms consistent with pancreatitis upon enrollment. It is unclear whether the pancreatitis was caused by the investigational agent, her known pancreatic disease, or a combination of the two. The fourth patient received 1 dose of pegaspargase. She was admitted on cycle 1 day 10 at her local hospital with shortness of breath and found to have worsening pleural effusions bilaterally. Pulse oximetry showed consistent desaturation to $90 \%$. Thoracentesis was complicated by a right-sided pneumothorax requiring chest tube insertion. Subsequently, bilateral Pleurex catheters were placed with satisfactory control of her effusions and normalized oxygenation. She remained hospitalized and on 
the seventh day of hospitalization had a respiratory arrest with pulseless electrical activity. Resuscitation was unsuccessful. Admission clotting studies were normal; bilateral lower extremity Doppler ultrasound had been performed on day 2 showing no evidence of lower extremity DVT and admission 12-lead EKG showed no evidence of coronary ischemia. Autopsy was not performed; therefore, the cause of the arrest was not defined. Pulmonary embolism was suggested by her home physicians as consistent with her clinical picture immediately prior to the arrest.

\section{Discussion}

Repurposing known agents for new applications is a method of augmenting the therapeutic armamentarium for ovarian cancer patients. Our recent preclinical data identified new anti-adhesive and anti-angiogenic activities of L-ASP leading to the hypothesis that L-ASP may be effective against solid tumors, such as ovarian cancer. Authors of this study as well as other investigators have shown that decreased ASNS levels in ovarian cancer cell lines and fresh tumor specimens may lead to increased susceptibility to L-ASP. Moreover, L-ASP inhibits capillary-like tube formation of HMVECs and decreases heterotypic adhesion between ovarian cancer and endothelial cells (9-12). This clinical trial was designed to translate preclinical findings with asparaginase into clinical practice and evaluate the efficacy of pegaspargase in advanced ovarian cancer patients. Four patients were treated with $2000 \mathrm{IU} / \mathrm{m}^{2}$ pegaspargase during this clinical trial and enrollment was discontinued early due to reaching predefined safety limits.

The extent and frequency of AEs in our study patients (4 out of 4 , including 1 mortality) was higher compared to those reported by previous studies on the use of L-ASP or pegaspargase on solid tumors. A phase I dose-escalation study of pegaspargase in 28 adult patients with advanced solid tumors reported no treatment-related patient mortality and only 1 case of grade 4 allergic reaction (11). Three patients (11\%) developed grade $\leq 3$ allergic reactions. Grade 2 and 3 elevations of amylase and lipase occurred and were neither dose-related nor associated with symptoms. No patients developed clinical pancreatitis. Grade 1 and 2 elevations of PT and/or aPTT levels, as well as decreases in fibrinogen, were observed but not associated with clinical signs or symptoms. Two patients with advanced malignant melanoma developed lower extremity DVT in a setting of normal coagulation parameters. The side effects most consistently associated with increasing dose of pegaspargase included nausea/vomiting, fatigue/weakness and anorexia/weight loss. All patients treated with the dose used in our study $\left(2,000 \mathrm{IU} / \mathrm{m}^{2}\right.$ i.m. every 2 weeks) experienced some degree of fatigue or weakness. Sixty percent of patients on that trial developed nausea/vomiting, with $20 \%$ having grade 3 anorexia or weight loss. The investigators concluded that pegaspargase was generally well-tolerated in patients with advanced solid tumors and that a dosage of $2,000 \mathrm{IU} / \mathrm{m}^{2}$ by i.m. injection every 2 weeks resulted in significant depletion of serum L-asparagine. It has not been elucidated whether it was our i.v. administration that led to increased toxicity.

The toxicities observed in our study were not unexpected for pegaspargase therapy. Venous thrombosis has been reported in patients with solid tumors and hematological malignancies treated with pegaspargase and L-ASP $(11,18)$. Associations between anti-thrombin III deficiency and venous thromboembolism in patients treated with L-ASP have been reported $(19,20)$. Anti-thrombin III levels were not measured in the patient who died during the study; however, PT and aPTT were within normal limits upon her terminal admission and no lower extremity DVTs were noted on Doppler ultrasound $\sim 1$ week prior to her death. Other significant toxicities noted in our study, such as pancreatitis, fatigue, nausea, anorexia and a grade 3 allergic reaction, were consistent with those noted in previous studies $(11,13,14)$. We would not recommend further investigation of pegaspargase in patients with advanced ovarian cancer, due to the incidence and severity of adverse effects in our study population.

\section{Acknowledgements}

This study was supported by the Intramural Program of the Center for Cancer Research, National Cancer Institute, USA.

\section{References}

1. Jemal A, Siegel R, Ward E, Hao Y, Xu J and Thun MJ: Cancer statistics, 2009. CA Cancer J Clin 59: 225-249, 2009.

2. Liotta LA and Kohn EC: The microenvironment of the tumour-host interface. Nature 411: 375-379, 2001.

3. Hollingsworth HC, Kohn EC, Steinberg SM, Rothenberg ML and Merino MJ: Tumor angiogenesis in advanced stage ovarian carcinoma. Am J Pathol 147: 33-41, 1995.

4. Cannistra SA, Matulonis UA, Penson RT, et al: Phase II study of bevacizumab in patients with platinum-resistant ovarian cancer or peritoneal serous cancer. J Clin Oncol 25: 5180-5186, 2007.

5. Capizzi RL, Bertino JR, Skeel RT, et al: L-asparaginase: clinical, biochemical, pharmacological, and immunological studies. Ann Intern Med 74: 893-901, 1971.

6. Clarkson B, Krakoff I, Burchenal J, et al: Clinical results of treatment with $E$. coli $\mathrm{L}$-asparaginase in adults with leukemia, lymphoma, and solid tumors. Cancer 25: 279-305, 1970.

7. Haskell CM, Canellos GP, Cooney DA and Hansen HH: Biochemical and pharmacologic effects of L-asparaginase in man. J Lab Clin Med 75: 763-770, 1970.

8. Haskell CM, Canellos GP, Leventhal BG, et al: L-asparaginase: therapeutic and toxic effects in patients with neoplastic disease. N Engl J Med 281: 1028-1034, 1969.

9. Lorenzi PL, Llamas J, Gunsior M, et al: Asparagine synthetase is a predictive biomarker of L-asparaginase activity in ovarian cancer cell lines. Mol Cancer Ther 7: 3123-3128, 2008.

10. Lorenzi PL, Reinhold WC, Rudelius M, et al: Asparagine synthetase as a causal, predictive biomarker for L-asparaginase activity in ovarian cancer cells. Mol Cancer Ther 5: 2613-2623, 2006.

11. Taylor CW, Dorr RT, Fanta P, Hersh EM and Salmon SE: A phase I and pharmacodynamic evaluation of polyethylene glycol-conjugated L-asparaginase in patients with advanced solid tumors. Cancer Chemother Pharmacol 47: 83-88, 2001.

12. Yu M, Henning R, Walker A, et al: L-asparaginase inhibits invasive and angiogenic activity and induces autophagy in ovarian cancer. J Cell Mol Med 16: 2369-2378, 2012.

13. Graham ML: Pegaspargase: a review of clinical studies. Adv Drug Deliv Rev 55: 1293-1302, 2003.

14. Ho DH, Brown NS, Yen A, et al: Clinical pharmacology of polyethylene glycol-L-asparaginase. Drug Metab Dispos 14: 349-352, 1986.

15. Simon R: Optimal two-stage designs for phase II clinical trials. Control Clin Trials 10: 1-10, 1989.

16. Therasse P, Arbuck SG, Eisenhauer EA, et al: New guidelines to evaluate the response to treatment in solid tumors. European Organization for Research and Treatment of Cancer, National Cancer Institute of the United States, National Cancer Institute of Canada. J Natl Cancer Inst 92: 205-216, 2000. 
17. Rose PG, Tian C and Bookman MA: Assessment of tumor response as a surrogate endpoint of survival in recurrent/ platinum-resistant ovarian carcinoma: a Gynecologic Oncology Group study. Gynecol Oncol 117: 324-329, 2010.

18. Guarnaccia C, Fabbiano F and Mancuso L: Pulmonary embolism and L-asparaginase therapy. Acta Haematol 71: 215, 1984.
19. Pitney WR, Phadke KP and Dean S: Antithrombin III deficiency during asparaginase therapy. Lancet 1: 493-494, 1980.

20. Vellenga E, Mulder NH and Nieweg HO: Antithrombin III deficiency during asparaginase therapy. Lancet 1: 649-650, 1980. 